\title{
Prevención del abuso y la explotación sexual comercial: Un proyecto exitoso en la zona sur de Costa Rica
}

\author{
Marcela Sanabria Hernández ${ }^{1}$ y Georgina Marín Gálvez ${ }^{2}$
}

1. Máster en Evaluación de Programas y Proyectos de Desarrollo por la Universidad de Costa Rica (UCR) y Psicopedagogía por La Salle. Actualmente, encargada de la Cátedra de Psicopedagogía de la UNED. Correo electrónico: msanabria@uned.ac.cr

2. Máster en Psicopedagogía por la UNED y licenciada en Psicología. Actualmente encargada de la Cátedra de Psicología Educativa de la UNED. Correo electrónico: gmaring@uned.ac.cr

Recibido: Febrero 2014 • Aceptado: Mayo 2014

\section{RESUMEN}

El presente artículo tiene como objetivo dar a conocer el proyecto que ejecutaron la Escuela de Ciencias Sociales y Humanidades y el Centro Universitario de Ciudad Neily (ambos de la Universidad Estatal a Distancia, UNED) desde el 2007 hasta el 2012, para la prevención del abuso y la explotación sexual comercial en la población menor de edad de la zona sur de Costa Rica. Este proyecto se gesta con la intención de dar respuesta a una necesidad particular que se ha identificado en la zona, como es la atención a las diversas manifestaciones de abuso, principalmente sexual y de explotación sexual comercial infantil.

De igual forma responde a una intencionalidad planteada en el quehacer propio de la Universidad de vincularse con las regiones, así como el acompañamiento y atención a diversas problemáticas que puedan ser abordadas desde la experiencia universitaria.

El proyecto inicia en el año 2007 con el desarrollo de talleres sobre la temática del abuso y la explotación sexual con una población de estudiantes de cuarto, quinto y sexto grados de la educación general básica, en escuelas de los cantones de Golfito y Corredores; y con recursos de fondos del Consejo Nacional de Rectores (CONARE). Posteriormente, los talleres se trabajaron con población adolescente.

Paralelo a la realización de estos talleres se produjeron programas de radio transmitidos por una emisora cultural de la zona. Dichos programas fueron elaborados por un grupo de jóvenes que se conformó como producto de los talleres antes mencionados.

Para los años 2008, 2009 y 2010 las experiencias continuaron básicamente con la realización de talleres y los grupos juveniles; se incorporaron los cines foros y las charlas a padres y madres de familia. También, en este período se inicia un trabajo en los centros educativos para capacitar al personal docente y administrativo.

Entre el 2011 y el 2012, con fondos de la Comisión Regional Interuniversitaria CRI- CONARE, se implementa el proyecto con el nombre "Formación Integral de Calidad para las Comunidades Educativas de la zona sur", realizado en vinculación con la Universidad de Costa Rica y la Universidad Nacional.

A partir de todas estas experiencias y al determinar el interés de las comunidades, se decide llevar a cabo el Proyecto Intercátedras que permitiera conjuntar el accionar de diferentes cátedras de la Escuela de Ciencias Sociales y Humanidades en una temática en común, proyectada a nivel nacional y con la participación además del estudiantado.

El recorrido por estos seis años de experiencias acercándose al tema del abuso y la explotación sexual comercial infantil permite identificar la necesidad de que el tema de la protección a la niñez debe ser una constante en nuestro quehacer como académicos y más aún en una escuela con una visión humana y social. 
Pero esta realidad no puede quedar únicamente en el ámbito universitario, se debe gestionar para que los resultados de dichos proyectos puedan ser expuestos ante otras instancias y puedan servir de insumos a las políticas que en materia de niñez y adolescencia debe desarrollar este país.

Palabras clave: explotación sexual comercial infantil (ESCI), zona sur, prevención, abuso, proyecto exitoso.

\begin{abstract}
This article aims to present the project that has been running the School of Social Sciences and Humanities and the University Center of Ciudad Neily (both from the Universidad Estatal a Distancia, UNED), from 2007 to 2012, for the prevention of abuse and commercial sexual exploitation in the minor population of the Southern Zone of Costa Rica.

This project unfold with the intention of responding to a particular need has been identified in the area, as is attention to the various forms of abuse, particularly sexual and commercial sexual exploitation.

Similarly responds to intentionality raised in the proper work of the University as its relationship with the regions as well as the support and attention to various problems that can be addressed from the college experience.

The project started in 2007 with the development of workshops on the subject of the abuse and sexual exploitation with a student population 4, 5 and 6 grade of basic general education in schools in the cantons of Golfito and Corredores ; funds and resources of the National Council of Rectors (CONARE) . Subsequently the workshops worked with adolescents.

Parallel to these workshops were produced radio programs broadcast by a radio culture of the area . These programs were developed by a group of young people was formed as a result of the above workshops.

For the years 2008, 2009 and 2010 remain basically experiences with workshops and youth groups ; theaters forums and talks to parents and mothers are incorporated.

Also in this period work in schools began training faculty and staff.

Between 2011-2012 and with funding from the Inter- Regional Commission CRI- CONARE , the project named "Integrated Quality Training for Educational Communities of the Southern Zone " held in association with the University of Costa Rica and is implemented National University . From all these experiences and to determine the interest of the communitie, it was decided to carry out the project Intercátedra ; that would combine the activities of different Departments of the School of Social Sciences and Humanities in a common theme, designed at national level and also with the partition of the student.

The journey through these 6 years of experience approaching the issue of child abuse and commercial sexual exploitation, to identify the need for the issue of child protection must be a constant in our work as academics and more in a school with human and social vision.

But this reality can not be left solely at the university level, must be managed so that the results of such projects can be exposed to other instances and can serve as inputs to policies relating to children and adolescents must develop this country.
\end{abstract}

Key words: commercial sexual exploitation of children, South Zone, prevention, abuse, successful project.

\section{Introducción}

Teniendo presente la exhortación que hace la Universidad Estatal a Distancia (UNED) de trabajar por los derechos humanos en procura de construir una sociedad cada vez más justa es que la Escuela de Ciencias Sociales y Humanidades (ECSH) en forma conjunta con el Centro Universitario de Ciudad
Neily desarrollaron una serie de proyectos e iniciativas enmarcadas en la temática de un trabajo preventivo en favor de la niñez y de la adolescencia de la zona sur del país; principalmente de los cantones de Corredores y de Golfito. El presente artículo ofrece una revisión temática de los principales conceptos que se consideran en el abordaje de la explotación sexual comercial infantil, así como la referencia a 
estudios nacionales que acompañan las iniciativas que se gestan desde las instancias rectoras en materia de niñez y adolescencia.

Por otra parte, se presenta la trayectoria de todas las acciones llevadas a cabo por la $\mathrm{UNED}^{3}$ entre los años 2007 y 2011 mediante la ruta del proyecto, definida en las siguientes etapas:

- 2007 al 2008: "Primeros Pasos"

- 2009 al 2010: "Proyectándose a la comunidad"

- 2011 al 2012: "Fortaleciendo el proceso"
Para cada una de estas etapas se establece una síntesis del proyecto implementado, la población meta a la cual estuvo dirigida, qué se logró abarcar y los productos que resultaron de dichas acciones.

Al final, el artículo establece un análisis en forma de lecciones aprendidas desde los ámbitos de la visión propia de los niños, las niñas y adolescentes sobre su realidad; y de las personas gestoras del proyecto, quienes terminan haciendo su propio proceso de desmitificación ante lo atroz de la temática y así reconceptualizando la necesidad de trabajar con aquellas personas, instituciones o

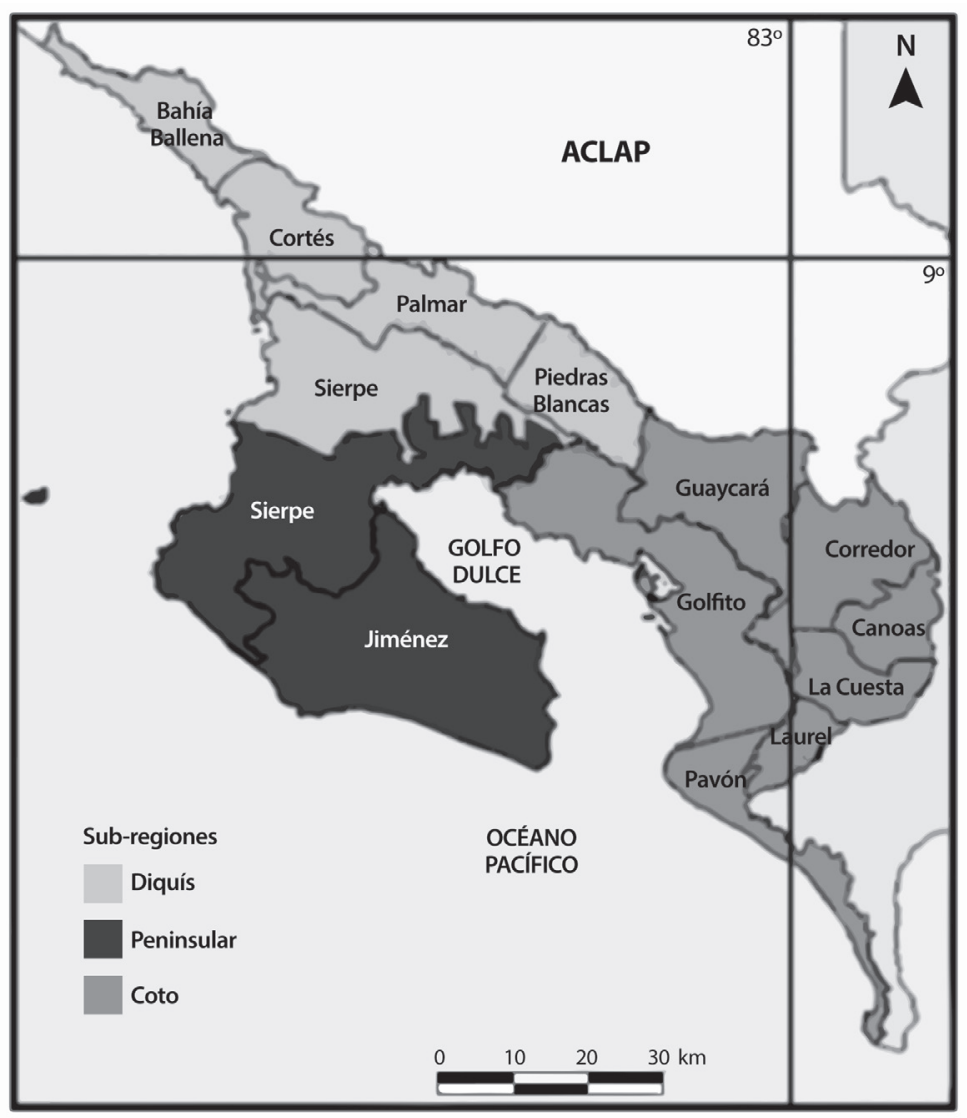

Figura 1. Acosa (2001). Subregiones administrativas y políticas. Áreas de conservación Osa. Recuperado de <https://www.inbio.ac.cr/ecomapas/acosau/generalidades.htm>

3. Representada por la ECSH y el Centro Universitario de Ciudad Neily. 
instancias que se vuelven protectoras de una población tan vulnerable.

De igual forma se incluye un apartado de conclusiones, donde se rescata como idea principal la importancia que tiene el poder implementar experiencias que atiendan necesidades particulares (como el tema en cuestión), como parte de los proyectos de extensión que desarrollan las universidades.

\section{Antecedentes}

Como se mencionó en el apartado anterior, la UNED ha venido desarrollado acciones mediante la implementación de proyectos en favor de la niñez y de la adolescencia en la zona sur (cantones de Corredores y Golfito) del país desde el año 2007, en coordinación con el Centro Universitario de Ciudad Neily.

Como se puede observar en la figura de la página anterior, Corredores y Golfito son cantones de la provincia de Puntarenas (Región Brunca), que se ubican en la zona sur del país cerca de la frontera con Panamá. El cantón de Corredores tiene por cabecera a Ciudad Neily.

Ambos cantones han sido catalogados como zonas de gran riqueza natural pero al mismo tiempo lugares donde priva la pobreza, la vulnerabilidad infantil y condiciones que atentan contra el desarrollo humano integral.

Tal y como lo indican Arias y Sánchez (2010: 27) la región presenta altos niveles tanto de pobreza como de pobreza extrema; así como también de analfabetismo (p. 41), esto incide en forma directa y pone en un alto riesgo de vulnerabilidad a la población menor de edad.

Según indica Claramunt, la explotación sexual comercial es considerada por la OIT como un delito asimilable a la esclavitud y al trabajo forzoso (2005: 4) y una de las peores formas de trabajo infantil; por ende considerado un flagelo para una población que requiere de atención constante y directa en materia de derechos.

Aunado al tema de la explotación sexual comercial infantil es necesario hacer referencia a las vivencias de abuso sexual que sufre la población menor de edad, las cuales si bien es cierto no solo responden a poblaciones rurales sí tienden a ser entendidas en cierto grado de "normalidad" (en estas zonas) basadas en las conductas patriarcales y adultocéntricas que las justifican.

El abuso sexual infantil es considerado una forma de maltrato infantil que atenta contra la dignidad del ser humano y sus efectos inciden en aspectos biológicos, psicológicos, sociales y emocionales. Este puede ser conceptualizado como la utilización de un menor de edad para la gratificación sexual de una persona adulta o grupo de adultos (Lago y Céspedes, 2005: 16). En el caso de que el abuso sea llevado a cabo por un familiar con un cierto grado de consanguineidad el acto es conocido como incesto.

Es importante tener presente que en caso de la explotación sexual la persona menor de edad es utilizada con fines comerciales; comprende por ende la pedofilia, el tráfico para turismo sexual o la pornografía a través de Internet, entre otros (Lago y Céspedes, 2005: 17).

En Costa Rica, se han llevado a cabo acciones desde diversos ámbitos tanto gubernamentales como privados que permiten crear un frente común para mitigar o minimizar en su máxima expresión esta plaga que atenta contra una de las mayores riquezas de la sociedad costarricense como es su población menor de edad.

Algunas de estas iniciativas es la consolidación de la Comisión Nacional contra la Explotación Sexual Comercial (CONACOES), creada desde 1996; la Ley contra la explotación sexual comercial (7899) y la Ley de Fortalecimiento de esta lucha (Ley 7594) (2007). Se evidencia de esta manera el interés de que tiene Costa Rica por constituirse como un país que vela por los derechos de su población menor de edad, tanto desde lo preventivo como en lo punitivo.

\section{Ruta del proyecto}

El proyecto desde sus inicios opta por el trabajo preventivo contra el abuso y la explotación sexual comercial mediante talleres y actividades educativas con niñez y adolescencia. 


\section{Primeros pasos (2007-2008)}

La Escuela de Ciencias Sociales y Humanidades de la Universidad Estatal a Distancia (UNED), en febrero del 2007, con recursos económicos de los Fondos del Consejo Nacional de Rectores (CONARE) inicia el trabajo contra la explotación sexual en la zona sur de Costa Rica.

$\mathrm{Al}$ conocer la zona y sus necesidades, emergen fuertemente arraigados los elementos históricos que la caracterizan como una región que ha tenido que enfrentar grandes cambios a nivel productivo y por ende a nivel social y económico. Una consecuencia de estos cambios ha sido la falta de oportunidades educativas y laborales vinculadas con la estancia y salida de la actividad bananera y la ausencia de una acertada estrategia de desarrollo alternativo para la región (OIT, 2005).

Con el paso del tiempo han surgido nuevas opciones de desarrollo en la zona, tales como el turismo, con la creación de nuevas marinas y la infraestructura hotelera que dicha actividad requiere. Sin embargo, la nueva oportunidad trae también sus retos, pues se puede tender a promocionar el turismo sexual y así perpetuar las raíces históricas que tanto daño y dolor han causado a la población femenina y a la niñez.

Precisamente al considerar la situación de vulnerabilidad en la que vive la niñez y la adolescencia, emerge la necesidad de un trabajo preventivo sistemático y generador de procesos constructivos de habilidades protectoras en esta población. Con este fin, el proyecto incluyó trabajo con niños y niñas estudiantes de cuarto, quinto y sexto grado de algunas escuelas de la zona. También la formación de un grupo juvenil dirigido a personas que no estaban insertas en el sistema educativo formal.

En mayo del 2007, se trabajó con una población de 867 niños, niñas y adolescentes en edad escolar. La metodología utilizada consistió en una serie de talleres quincenales con cada grupo. Se utilizó la técnica del radio foro, de manera que se hizo una selección de programas radiales especializados para la población menor de edad, con temas orientados a desarrollar el conocimiento de sí mismos, su autoestima, la capacidad de tomar decisiones, las habilidades para la vivencia de la amistad y la convivencia grupal. También se desarrollaron temas como prevención del abuso, la explotación sexual comercial y la drogadicción. El diseño de cada taller incluyó actividades lúdicas, materiales y encuestas que en su momento permitieron conocer la realidad que vive dicha población en relación con la temática del taller. Al finalizar, una evaluación para que los participantes expresaran su opinión sobre la vivencia del taller.

La experiencia para el año 2008 se continuó con una población de 562 niños, niñas y preadolescentes de las escuelas participantes. Y se trabajó igualmente con talleres quincenales para cada grupo, cuyo tema principal fue el código de los derechos de la niñez y la adolescencia. Se considera que el conocimiento de este permite a los menores de edad defenderse de diversos abusos incluyendo la explotación sexual comercial.

Para el desarrollo del presente proyecto, se contó con el apoyo de varias instituciones de la zona, como la Sede Regional del Ministerio de Educación Pública, que brindó espacios y horarios de trabajo con grupos de las escuelas que a continuación se mencionan: Licenciado Alberto Echandi, Río Nuevo, Central de Coto 47, Santa Marta, Líder de Paso Canoas y Excelencia Confraternidad.

Con respecto al trabajo con los jóvenes se creó el Grupo Conexión Juvenil dirigido a adolescentes del cantón de Corredores que no estaban inscritos en el sistema educativo formal. Se les convocó a participar en el grupo por medio de un anuncio radial transmitido en la Emisora Cultural de Corredores. El grupo se inició con 12 jóvenes, tres de ellos provenientes de la zona indígena. El objetivo del trabajo fue siempre en la línea de la prevención del abuso y la explotación sexual comercial y consistió en brindar capacitación básica para la elaboración de programas radiales. Se realizaron talleres para el desarrollo de habilidades de investigación, elaboración de guiones radiales, manejo e impostación de la voz, elementos básicos para planificación y realización de entrevistas, así como talleres de aspectos 
más técnicos como uso de grabadoras, cámaras fotográficas y producción de los programas radiales. Además, se realizaron talleres de desarrollo personal en temas como autoestima, amistad, sexualidad y valores. El trabajo grupal permitió construir el nombre del grupo y optar porque los programas radiales tuvieran como población meta a los jóvenes, así como definir los temas de mayor interés para dicha población. Conexión Juvenil produjo en el 2007 seis programas radiales. En el 2008, se continuó el proceso formativo mediante los talleres y se logró grabar y editar una nueva serie de 10 programas radiales. Todos los programas fueron transmitidos mediante la Emisora Cultural de Corredores en horarios accesibles para la audiencia juvenil de la región.

En todo este proceso con el grupo Conexión Juvenil se contó con el apoyo técnico del Programa Onda UNED y de la Emisora Cultural de Corredores.

En Puerto Jiménez la Asociación Rescate de Valores (asociación de índole religiosa, cuyo objetivo es promover temas relacionados con valores en dicha comunidad) solicitó que el proyecto se desarrollara para la comunidad y se encargó de reunir tanto a niños, niñas y adolescentes como también a algunos adultos.

De esta manera, siempre en el marco de la prevención del abuso y de la explotación sexual comercial, en Puerto Jiménez se dio inicio a una serie de talleres con temas formativos dirigidos a un grupo de 12 integrantes. Además, se desarrollaron talleres dirigidos a un grupo de 18 personas adultas, entre ellas líderes de la comunidad y personal administrativo de los centros educativos. La meta propuesta fue fortalecer el liderazgo de estas personas para que promuevan el respeto de los derechos de la población menor de edad y que como líderes contribuyan a la erradicación tanto del abuso como de la explotación sexual.

Todo el trabajo realizado en el marco de esta iniciativa fue planeado en concordancia con los objetivos propuestos por la Comisión $\mathrm{Na}$ cional contra la Explotación Sexual Comercial (CONACOES). Además, es importante resaltar la vinculación en este proceso con la Universidad
Nacional, cuyo aporte consistió en la realización del proyecto Deserción Cero como camino preventivo para eliminar de raíz este tipo de problemática que aqueja a la sociedad.

\section{Proyectándose a la comunidad (2009-2010)}

El 2009 permitió consolidar el trabajo preventivo con 306 niños, niñas y adolescentes siempre por medio de talleres y actividades educativas de tipo lúdico.

El grupo juvenil anteriormente denominado Conexión Juvenil creció con nuevos integrantes y por decisión de sus miembros decidieron llamarlo Metamorfosis, nombre que simbolizó el proceso de crecimiento que ellos estaban viviendo. Se continuó el proceso formativo y de capacitación mediante talleres. Y se logró que Metamorfosis tuviera presencia en actividades como ferias científicas y rendición de cuentas, espacios en los que divulgaron la importancia de los derechos de la niñez y la adolescencia, así como el riesgo que representa tanto el abuso como la explotación sexual comercial infantil. Durante este año la producción radial fue de 14 programas, igualmente transmitidos por la Emisora Cultural de Corredores.

Al mismo tiempo, el trabajo en la comunidad de Puerto Jiménez permitió el desarrollo de varios cineforos con una nutrida participación de 35 personas. Los temas abordados giraron siempre en torno a crecimiento personal y a los derechos de los niños, las niñas y adolescentes, conscientes de que estos procesos brindan las herramientas necesarias para la protección de dicha población.

Otro aspecto del trabajo realizado fue el que se desarrolló con 10 familias de Ciudad Neily, en las que se pudieron identificar 29 casos de niños, niñas y adolescentes en situaciones de vulnerabilidad por el alto índice de pobreza, la desintegración familiar, el desempleo y la poca escolaridad. Con estas personas fue de vital importancia desarrollar ciertas habilidades para que los menores de edad contaran con mayor protección y cuidado por parte de sus familias. 
Para el año 2010 el grupo juvenil Metamorfosis alcanzó un total de 30 participantes, lo cual facilitó el incremento de su presencia en actividades tanto de la comunidad como de la UNED, siempre con el lema de promover la prevención del abuso y la explotación sexual comercial. Además, se logró brindar espacios formativos con temas inherentes al desarrollo afectivo y sexual propio de esta edad. Temas que también se aprovecharon en la producción de catorce nuevos programas radiales.

A raíz del trabajo realizado en la zona sur y de la magnitud del problema del abuso y de la explotación sexual comercial, surgió también la inquietud de incorporar al alumnado de la UNED en esta lucha, mediante la iniciativa del Proyecto Intercátedras, con el lema "Acerquemos a nuestros estudiantes a la prevención del abuso y la explotación sexual comercial", trabajo conjunto que se gestó entre las cátedras de Historia, Trabajo Social, Formación Cívica y Geografía, Psicología Educativa y Turismo Sostenible de la Escuela de Ciencias Sociales y Humanidades. El objetivo principal consistió en facilitar la participación del estudiantado en el quehacer social de la Universidad, sensibilizarlo ante la problemática del abuso y la explotación sexual comercial infantil, crear un compromiso en la lucha contra esta esclavitud y de igual forma promover líneas de investigación en la institución.

Para el logro del objetivo anterior se asignó a los estudiantes de la UNED, como parte de algunas asignaturas, una tarea, investigación o actividad que pusiera en contacto al alumnado con la realidad del problema desde las diversas áreas: el abuso y la explotación sexual comercial infantil desde el punto de vista histórico, sus consecuencias desde el punto de vista psicológico, la labor preventiva vista desde la óptica del trabajo social y dentro del proceso de la formación cívica, así como el papel del turismo sostenible en la implementación o en la erradicación de este flagelo.

Luego de cuatro años, en el 2010, la iniciativa cierra una etapa culminante de su vida. El trabajo realizado, las metas alcanzadas y los productos obtenidos se pueden resumir en los cuadros 1 y 2 .

Como producto de esta etapa y para la divulgación de resultados, se elaboró la exposición fotográfica denominada Nuevos Horizontes de Esperanza, y su respectivo catálogo, con el fin de que se presente en las diversas regiones del país, ya sea por solicitud explícita de alguna institución o bien por alguna actividad o celebración inherente al tema.

\section{CUADRO 1}

Resumen de componentes del proyecto (2007-2010)

\begin{tabular}{|c|c|c|c|}
\hline Población & $\begin{array}{l}\text { Cantidad de } \\
\text { personas }\end{array}$ & Talleres & Impacto \\
\hline 17 Escuelas & $\begin{array}{l}2169 \\
\text { Niñas, Niños y } \\
\text { Adolescentes }\end{array}$ & 610 Talleres & $\begin{array}{l}\text { Explotación sexual comercial visibilizada como } \\
\text { problema } \\
\text { Desarrollo de actitudes protectoras } \\
\text { Mejor autoestima y crecimiento en valores } \\
\text { Desarrollo del sentido de pertenencia }\end{array}$ \\
\hline $\begin{array}{l}\text { Directores } \\
\text { Docentes }\end{array}$ & $\begin{array}{l}10 \text { Directores } \\
260 \text { Docentes }\end{array}$ & 8 Capacitaciones & $\begin{array}{l}\text { Mayor sensibilización ante la ESC } \\
\text { Mejoramiento en la calidad educativa } \\
\text { Preparación para comprender el desarrollo } \\
\text { psicosexual de la población adolescente } \\
\text { Mayor claridad en los procesos de denuncia }\end{array}$ \\
\hline
\end{tabular}


CUADRO 1 (Continuación...)

Resumen de componentes del proyecto (2007-2010)

\begin{tabular}{|c|c|c|c|}
\hline Población & $\begin{array}{l}\text { Cantidad de } \\
\text { personas }\end{array}$ & Talleres & Impacto \\
\hline $\begin{array}{l}\text { Grupo Radio } \\
\text { Conexión Juvenil } \\
\text { Metamorfosis }\end{array}$ & 62 Adolescentes & $\begin{array}{l}63 \text { Sesiones de } \\
\text { trabajo } \\
44 \text { Programas de } \\
\text { radio }\end{array}$ & $\begin{array}{l}\text { Adolescentes con mejor percepción de sí mismos } \\
\text { Visión integral de la sexualidad } \\
\text { Habilidades para investigar temas de su interés } \\
\text { Habilidades para la producción de programas } \\
\text { de radio }\end{array}$ \\
\hline $\begin{array}{l}\text { Comunidad } \\
\text { Puerto Jiménez }\end{array}$ & 80 personas & $\begin{array}{l}20 \text { Sesiones de } \\
\text { trabajo }\end{array}$ & $\begin{array}{l}\text { Proceso de sensibilización ante la ESC } \\
\text { Metodología para la prevención de la ESC }\end{array}$ \\
\hline $\begin{array}{l}\text { Guías de turismo } \\
\text { Local }\end{array}$ & 60 personas & 2 sesiones de trabajo & $\begin{array}{l}\text { Guías comprometidos en la lucha contra la ESC } \\
\text { Desarrollo de habilidades protectoras para sí } \\
\text { mismos y sus empresas }\end{array}$ \\
\hline
\end{tabular}

Fuente: Marín, Castillo y Villalobos (2010).

CUADRO 2

Resumen del Proyecto Intercátedras (2010)

\section{Proyecto: Acerquemos a Nuestros Estudiantes a la Prevención del Abuso y la Explotación Sexual Comercial}

Actividad Impacto

Proyecto Intercátedras: Acerquemos a Nues-

600 futuros docentes comprometidos en la lucha contra la tros Estudiantes a la Prevención del Abuso y la explotación sexual comercial en niños, niñas y adolescentes. Explotación Sexual Comercial

Participación activa en la CONACOES de Ciudad Neily

UNED posicionada como fuerza viva en la comunidad. Aporte de talleres y capacitaciones vinculados con la red local contra la violencia.

Proceso de elaboración y publicación de la exposición itinerante de fotografías del proyecto Divulgación de la experiencia como modelo y propuesta de trabajo preventivo.

titulada Nuevos Horizontes de Esperanza

Dicha exposición está disponible para presentarla donde se solicite.

Fuente: Marín, Castillo y Villalobos (2010). 


\section{Fortaleciendo el proceso (2011-2012)}

En el año 2011 se continuó el proyecto con el nombre de Formación Integral de Calidad para las Comunidades Educativas de la Zona Sur, realizado en vinculación con la Universidad de Costa Rica y la Universidad Nacional, siempre con el apoyo financiero de los fondos de la Comisión Regional Interuniversitaria (CRI- CONARE).

Este proyecto, desde la iniciativa trabajada por la UNED, se desarrolló a partir de los siguientes objetivos:

- Motivar y capacitar al estudiantado de los diferentes ciclos educativos acerca de la importancia de la educación como herramienta para su desarrollo y futuro económico y social y el logro de una ciudadanía plena, en el marco de la construcción de su proyecto de vida.

- Capacitar al personal docente y administrativo en servicio, en los diferentes niveles del sistema educativo, para la innovación pedagógica y el mejoramiento de los ambientes educativos, mediante temas como salud y

\section{CUADRO 3}

Ejecución de los Talleres en la Escuela

Lic. Alberto Echandi, sección 6-4 (2011)

\begin{tabular}{lcc}
\multicolumn{1}{c}{ Taller } & $\begin{array}{c}\text { Número } \\
\text { talleres }\end{array}$ & Población \\
\hline $\begin{array}{l}\text { Puedo ser lo que quiero } \\
\text { y me propongo }\end{array}$ & 4 & 84 \\
$\begin{array}{l}\text { Técnicas de estudio } \\
\text { Drogas, violencia, sexo y } \\
\text { presión social }\end{array}$ & 4 & 87 \\
Total & 4 & 92 \\
\hline
\end{tabular}

Fuente: A. Espinoza (2011) ambiente, interculturalidad, riesgo, género, derechos y desarrollo humano, promoviendo la contextualización y pertinencia de la política educativa.

- Intensificar las acciones con los padres y madres de familia para que, a su vez, contribuyan a incentivar la participación de sus hijos e hijas en el proceso educativo, favoreciendo su permanencia en este y disminuyendo la deserción o expulsión.

Para el logro de estos objetivos, se realizaron talleres con estudiantes de sexto y sétimo años, talleres con padres y madres de familia de estudiantes de sétimo año, reuniones con personal docente y administrativo de primaria y secundaria de la Escuela Alberto Echandi y del Liceo de Ciudad Neily, respectivamente.

Por otra parte, se realizó un diagnóstico para identificar la percepción sobre el proceso de articulación de primaria y secundaria que tiene el estudiantado de sexto grado y sétimo año.

De forma específica las acciones dieron los siguientes resultados.

\section{CUADRO 4}

Ejecución de los Talleres en el Liceo Académico de Ciudad Neily, sétimo año, sección 8 (2011)

\begin{tabular}{|c|c|c|}
\hline \multicolumn{3}{|c|}{$\begin{array}{c}\text { Ejecución de los Talleres } \\
\text { Liceo Académico de Ciudad Neily } \\
\text { Realizados con estudiantes de Sétimo sección } 8 \\
2011\end{array}$} \\
\hline Taller & $\begin{array}{l}\text { Número } \\
\text { talleres }\end{array}$ & Población \\
\hline $\begin{array}{l}\text { Puedo ser lo que quiero y } \\
\text { me propongo }\end{array}$ & 10 & 192 \\
\hline $\begin{array}{l}\text { Al cole, pura vida con mis } \\
\text { compas }\end{array}$ & 8 & 153 \\
\hline $\begin{array}{l}\text { Drogas, violencia, sexo y } \\
\text { presión social }\end{array}$ & 8 & 153 \\
\hline Total & 26 & 500 \\
\hline
\end{tabular}

Fuente: A. Espinoza (2011) 
También se realizaron cinco talleres sobre el desarrollo de la inteligencia emocional para el personal docente y administrativo, que abarcó una población de 150 participantes. Esta misma temática fue desarrollada en talleres para padres y madres de familia.

Para el equipo que ejecutó el proyecto fue de gran importancia conocer cómo la institución educativa se convierte en un factor protector para la prevención de la explotación sexual comercial de la población menor de edad. En ese sentido, el análisis de la información del diagnóstico realizado condujo a identificar esas variables que habilitan al centro educativo como un espacio apto para el crecimiento y desarrollo integral de los niños, niñas y adolescentes.

Toda esta información ha quedado documentada en el artículo "Análisis de la percepción que tienen los y las estudiantes de sexto y sétimo año, sobre su ingreso a la educación secundaria" (Marín y Sanabria, 2013). Dicho artículo evidencia lo importante que es, para la niñez y la adolescencia de estos distritos, la institución educativa. Para el estudiantado, está muy claro el deseo de asistir a la secundaria, porque esta les ofrece un espacio de interacción social y de aprendizaje indispensable en su proyecto de vida.

Desde el 2011 y hasta la actualidad se le da seguimiento al proyecto por iniciativa de diversas cátedras de la Universidad Estatal a Distancia (UNED) y con apoyo de la Rectoría, dado que no se contó con un presupuesto de parte de los fondos de la Comisión Nacional de Rectores para su continuidad.

En esta nueva etapa, el Proyecto Intercátedras busca involucrar al estudiantado y a los sectores administrativo y docente de la UNED con el objetivo de fomentar en estos el compromiso contra este tipo de esclavitudes, bajo el lema "Es asunto de todos y todas". Dicha iniciativa fue construida y realizada con la participación de la mayoría de las cátedras, programas y área de extensión de la Escuela de Ciencias Sociales y Humanidades, y contó con el apoyo de la Rectoría y del Programa Onda UNED.
Esta iniciativa permitió realizar por primera vez a nivel institucional la Celebración del Día Mundial Contra el Abuso y la Explotación Sexual Comercial en Menores de Edad y la Trata de Personas.

\section{Lecciones aprendidas}

El proceso de cinco años de trabajo preventivo del abuso y la explotación sexual comercial de las personas menores de edad en la zona sur deja innumerables lecciones aprendidas. Entre las más significativas, se citan:

\section{Desde la visión de la niñez y la adolescencia}

Cuando las niñas, los niños y adolescentes tienen la posibilidad de conocer el tema y comprenderlo como una de las esclavitudes modernas son capaces de emitir un criterio y de considerar acciones preventivas en favor propio y de sus iguales.

Tal como lo demuestran las palabras de un joven que participó en el proyecto en su época escolar, este debe ser un espacio permanente: "Lo he recomendado a unos amigos para que le ayuden, es una experiencia muy bonita y diferente, pienso que sería muy bueno para que ellos puedan aprender de las cosas o el mundo que los rodean, claro antes de que empiecen en el mundo de la maldad, fue muy bueno para mí" (encuesta realizada en 2010).

Además, resalta la importancia de contar con espacios juveniles que les permitan abordar temáticas de su interés en un marco de recreación, respeto y aceptación de sus opiniones. Esto se observa en la encuesta realizada, pues las personas participantes resaltan "que era un grupo de jóvenes unido para ayudar a los futuros jóvenes".

\section{Desde las personas gestoras del proyecto}

Uno de los aprendizajes ha consistido en reconocer la posición que la comunidad asume ante esta esclavitud, a la que considera como una práctica frecuente y parte de su cotidianidad. 
A partir de este reconocimiento, emerge la necesidad de trabajar esta temática desde una óptica diferente y apostando a la niñez y la adolescencia, las cuales tienen la posibilidad de generar un cambio social. De acuerdo con lo que plantea la UNICEF (2011), invertir en la niñez y la adolescencia implica que, desde el punto de vista social, se brinda la posibilidad de asegurar el cumplimiento de sus derechos y, por lo tanto, el bienestar y la equidad de esta población. Asimismo, para el país esta inversión social se proyecta en crecimiento económico y productivo de la región.

También se ha reconocido el potencial de la niñez y la adolescencia para convertirse en protagonistas de la lucha contra el abuso y la explotación sexual comercial, siempre y cuando medie un buen proceso de sensibilización. Prueba de ello es el aporte del grupo Metamorfosis y la participación de algunas de estas personas jóvenes en asociaciones o grupos cantonales que abordan la temática.

La institución educativa se convierte en un aliado en materia de prevención, siempre y cuando logre ser un espacio atractivo para la niñez y la adolescencia, esto quiere decir que ofrece las condiciones para su crecimiento integral.

El personal docente y administrativo de las instituciones educativas de la zona debe convertirse en un aliado estratégico en la prevención de estos flagelos. Este personal debe contar con la debida preparación para los procesos de denuncia y tratamiento de esta problemática, más aún cuando este personal se puede ver expuesto a represalias y amenazas que atentan contra su propia vida, su familia o sus pertenencias.

Otro aprendizaje tiene que ver con la radio como medio de comunicación, el cual se convirtió en un elemento fundamental para acercarse a la comunidad en una zona tan extensa y así difundir de manera creativa y atrayente mensajes preventivos contra la explotación sexual comercial.

La Universidad Estatal a Distancia, responde a una demanda social congruente con su misión que tiene un compromiso con la excelencia académica, el desarrollo de la cultura, la ciencia, el arte y los derechos humanos para la construcción de una sociedad justa y una cultura de paz (UNED, 2011).

\section{Conclusiones}

El recorrido histórico del proyecto Prevención del Abuso y la Explotación Sexual Comercial en Niños, Niñas y Adolescentes de la Zona Sur de Costa Rica, que luego dio lugar al Proyecto Intercátedras conocido como Es Asunto de Todos y Todas, permite delinear algunas conclusiones que a su vez se convierten en puntos de partida para nuevos campos de acción en favor de la niñez y la adolescencia y como retos pendientes que los futuros profesionales de la educación y en general de las Ciencias Sociales deben retomar.

La primera conclusión que se extrae de esta iniciativa es que el cuidado y la valoración de la niñez y la adolescencia y de sus derechos es una tarea pendiente en la sociedad costarricense. Debe recordarse que la niñez y la adolescencia en Costa Rica requieren mayor cuidado y apoyo tanto a nivel familiar y social como a nivel gubernamental.

Dicha tarea -que está ligada a la necesidad de romper cadenas históricas protegidas por grupos de interés, que se ven amenazados ante la toma de conciencia y el desarrollo integral de las personas menores de edad y sus familias- en parte se logra por medio del trabajo preventivo con iniciativas, programas o proyectos, tales como los ejemplos expuestos en este artículo.

Con respecto al tema de la prevención del abuso y la explotación sexual comercial en la niñez y la adolescencia, queda en evidencia que implica una lucha constante que debe involucrar a todos los sectores sociales, desde el gobierno hasta la familia, desde los educadores hasta los empresarios y por lo tanto es necesario emprender a nivel nacional un trabajo preventivo apostando por los niños, niñas y adolescentes mismos, quienes serán los que podrán impulsar desde sí una sociedad libre de este tipo de problemas.

De suma importancia es el promover el trabajo formativo con las personas adultas en temas como psicología del desarrollo infantil, derechos de la niñez y la adolescencia, entre 
otros, que permitan desarrollar relaciones interpersonales respetuosas y a su vez educativas con esta población.

Mayor atención se debe prestar a la preparación de los futuros padres y madres de familia a quienes corresponde forjar en sus hijos e hijas principios y valores y una sana autoestima que les permita protegerse de este tipo de flagelos.

También, es importante promover en la sociedad costarricense, la capacidad de defender los principios y valores y los derechos de los menores de edad mediante la denuncia. Crear y desarrollar esta cultura a su vez implicará la necesidad de sistemas de justicia con mayor capacidad de respuesta.

Analizando el proyecto como experiencia de la UNED, en tanto universidad a distancia, con una mediación pedagógica diferente, cabe resaltar la importancia que tiene la capacidad de reinventarse constantemente para encontrar la forma de sensibilizar e involucrar a sus estudiantes en la lucha contra este tipo de problemas sociales que aquejan a la niñez y adolescencia.

El Proyecto Intercátedras es un esfuerzo creativo, que además de involucrar a tantos menores de edad, convoca también a un número significativo de estudiantes y de académicos, lo que lo convierte en una exitosa experiencia formativa que permite integrar diferentes áreas del quehacer universitario, tales como la extensión, la investigación y la docencia.

Es importante resaltar también la figura del Centro Universitario de Ciudad Neily, en el cual se detecta la problemática del abuso y la explotación sexual en la zona y realiza las gestiones necesarias para impulsar la presente iniciativa. Aporta además el apoyo logístico y el acompañamiento constante a las gestoras en el trabajo realizado.

Dada la capacidad de la presente iniciativa de crear espacios de prevención, participación y formación en temas como el abuso y la explotación sexual comercial en la niñez y la adolescencia, marca una ruta por seguir y mejorar en la educación a distancia.

\section{Bibliografía}

Arias, R. y L. Sánchez (2010). "Análisis de la desigualdad socioeconómica en Costa Rica por criterio territorial". Informe final. Estado de la Nación. Recuperado de $<$ http://workspace.unpan.org/sites/Internet/Documents/ socioeconomica.pdf $>$.

Claramunt, C. (2005). "Explotación sexual comercial". Propuesta de trabajo para una atención integral a las personas menores de edad víctimas. OIT-IPEC. Recuperado de: <file:///C:/Users/Hnos/Downloads/2005_traff_comm_sexexpl_latinamer_costarica_es.pdf $>$.

EspinozA, A. (2011). Encuestas realizadas en las escuelas y colegio de la zona sur, en el marco de los proyectos CRICONARE. Inédito.

LaGo, G y J. Céspedes (2005). "Abuso sexual infantil". Ascofame. Recuperado de: <http://www.scp.com.co/precop/precop_files/modulo_5_vin_3/1630 $\% 20$ Abuso $\% 20$ infantil.pdf $>$.

Marín, G.; S. Castillo e I. Villalobos (2010). "Nuevos horizontes de esperanza: iniciativa Comunicación y recreación para la prevención del abuso y la explotación sexual comercial en niños, niñas y adolescentes en la zona sur, 2007-2010”. San José: UNED.

Orт (2005). “Tendencias mundiales del empleo. Informe sobre el empleo en el mundo: En 2004, ligero crecimiento del empleo, pero no suficiente". Revista Trabajo, n. ${ }^{\circ} 55$.

OIt/IPEC (2005). Documento de información básica sobre la problemática de la explotación sexual comercial de niños, niñas y adolescentes en Centroamérica, Panamá y República Dominicana. Recuperado de: <http://white. oit.org.pe/ipec/documentos/documento_basico_de_informacion_2005.pdf>.

UNICEF (2011). "Inversión en salud y protección social a la niñez y la adolescencia". Montevideo.

Universidad estatal a distancia (2011). Plan de Desarrollo Institucional: Para el Fortalecimiento de la Educación a Distancia. San José. 